\title{
Comparison of Mortality Rate Between Bipolar Hemiarthroplasty and Proximal Femoral Nail Anti-rotation for Intertrochanteric Fractures in Sanglah Hospital, Bali
}

I Wayan Suryanto Dusak, I Gusti Ngurah Wien Aryana, Cokorda Gde Oka Dharmayuda, I Wayan Subawa, Hans Kristian Nugraha, Made Arya Susila, Sri Mahadhana

Department of Orthopaedic and Traumatology Sanglah Hospital / Faculty of Medicine, University of Udayana, Bali, Indonesia

\section{Abstract}

Introduction: Intertrochanteric fractures occur in about $50 \%$ of all hip fracture events, with a mortality rate within 1 year after fracture reaching 15 to $20 \%$. The most common treatment nowadays is either the bipolar hemiarthroplasty procedure or proximal femoral nail anti-rotation (PFNA), although there is still no consensus regarding which is better from the two, especially on patient mortality.
\end{abstract}

Method: This study was an observational study using a retrospective cohort design. A total of 102 study subjects who met the inclusion requirements were grouped into 2 groups, one with bipolar hemiarthroplasty fixation treatment and another with PFNA fixation treatment. Mortality rate was recorded by survey 2 years after surgery.

Results: Chi-square test showed that 2-year mortality rate after intertrochanteric fracture treated with bipolar hemiarthroplasty $(21.4 \%)$ was significantly higher than the PFNA group $(10.3 \%)(p=0.028)$. Bipolar hemiarthroplasty group also had longer length of stay (50\%) than the PFNA group $(32.4 \%)$, albeit statistically insignificant $(p=0.13)$. There was no significant difference between the 2-year mortality rate and length of stay $(p=0.976)$.

Conclusion: Patients with intertrochanteric fractures who underwent bipolar hemiarthroplasty have significantly higher 2year mortality rate than similar patients underwent fixation with PFNA, while they did not experience higher length of stay than the PFNA group. Future prospective, multi center study with larger sample size will be likely to validate similar fixation choice needed to decrease the mortality rate in intertrochanteric fractures.

Keywords: Hip fracture; intertrochanteric fracture; bipolar hemiarthroplasty; proximal femoral nail anti-rotation; mortality Level of Evidence: III

\section{Article history}

Submitted: June $26^{\text {th }}, 2020$

Revise : July $6^{\text {th }}, 2020$

Accepted : July $7^{\text {th }}, 2020$
Corresponding Author: Cokorda Gde Oka Dharmayuda, MD. Department of Orthopaedic and Traumatology Sanglah Hospital / Faculty of Medicine, University of Udayana, Bali, Indonesia. email:cokdharmayuda76@gmail.com. 


\section{Introduction}

Intertrochanteric fractures occur in about $50 \%$ of all hip fracture events, with a mortality rate within 1 year after fracture reaching 15 to $20 \% .{ }^{1}$ It was projected that more than $50 \%$ of all hip fractures in the world will occur in Asia by 2050, mainly due to an increase in older population and longer life expectancy. In Japan, the risk of lifetime hip fractures for individuals at the age of 50 was reported to be $5,6 \%$ for men and $20 \%$ for women. In China, it is estimated that hip fracture cases will increase 6-fold from 0.7 million cases in 2013 to 4.5 million cases by $2050 .^{2}$

The ideal surgical technique for intertrochanteric fracture should allow the patient to regain mobility according to preoperative conditions, while also having the lowest intra and postoperative morbidity and mortality. Although proximal femoral nail-antirotation (PFNA) has been used extensively by orthopaedic specialists for patients with intertrochanteric fractures, PFNA failure has also been reported due to extensive comminution, osteoporosis, as well as cut out of the implant, femoral medialization, and lateral migration of proximal screws or helical blades. ${ }^{3,4}$ As a result, bipolar hemiarthroplasty which allows early full weight-bearing and avoiding the risk of osteosynthesis failure, has also been widely used as a satisfactory alternative for elderly patient with intertrochanteric fracture. ${ }^{5}$

Bipolar hemiarthroplasty indeed carries more surgical injuries in patients than PFNA, due to its longer surgical times and more blood loss. ${ }^{6}$ However, research by Dusak et al. of patients with intertrochanter femur fractures showed no significant difference from functional results measured by the Harris Hip Score, between the PFNA group (72) with bipolar hemiarthroplasty (69). ${ }^{7}$

The objective of this study was to compare the mortality rates and length of stay after bipolar hemiarthroplasty and PFNA fixation for intertrochanteric fracture, so that it could became a helpful basis for preoperative decision making in choosing between those two widely used treatments.

\section{Material and Methods}

This retrospective observational study was done in Sanglah General Hospital, using medical records of all patients with intertrochanteric fracture between January 2017 and December 2018. The subjects were then listed and their phone number collected. Survey were done by phone to confirm the data from medical records and to get the brief condition of the subjects.

The patients included in this study was male or female patients who had intertrochanteric femur fracture (as described by Boyd-Griffin Classification) and had undergone bipolar hemiarthroplasty or PFNA procedure in Sanglah General Hospital, could not walk with or without support, with physical status class III based on the American Society of Anaesthesiologists Classification (ASA Class) ${ }^{8}$ to minimize bias of comorbidity. The exclusion criteria was patients with history of neoplasm / malignancy and those who died during hospitalization postoperatively. The drop out criteria was patients with incomplete data in the medical record or patients who could not be reached by phone and those who died due to traffic accident. All of the patients were administered a preoperative intravenous injection of antibiotic ceftriaxone ( $2 \mathrm{~g}$ ), and general or spinal anaesthesia was used in all patients. Antibiotic treatments continued for 3 postoperative days. Rehabilitation through physical therapy was started as early as possible after surgery for all patients, and they were allowed to bear as much weight as they could tolerate.

The subjects were divided into two groups according to their treatment, the bipolar hemiarthroplasty group and the PFNA group. Subjects who died in this two year-period was noted and the total number of death in each group was divided by the number of subjects in each groups to obtain the mortality proportion.

They were also grouped according to their length-of-stay as another possible mortality predictor, whether it was less than 14 days, or equal to and more than 14 days. The length of stay was measured from the date of admission until the day of discharge from the hospital. Patients were discharged once they were in good general condition and able to ambulate under family supervision. Since length of stay itself might be 
attributed due to fixation choice, hence another comparison was also done.

The normality and homogeneity tests was performed by using the Kolmogorov-Smirnoff test and Levene test, respectively. The mortality proportion was then compared between each group by chi-square test because our data had normal distribution. Analysis by cross tabulation was performed for each group and the adjusted relative risk was calculated. Other variables measured in this study included age, sex, and intertrochanteric femur fracture type based on Boyd-Griffin classification. The descriptive analysis was also performed to the variables. The statistical analysis was performed by using SPSS Statistics 24 .

\section{Results}

In period between January 2017 until December 2018, there were 146 cases of intertrochanteric femur fracture in Sanglah General Hospital. After many exclusion and drop out, a total of 102 subjects were found, which consist of 34 subjects treated with bipolar hemiarthroplasty and 68 subjects treated with PFNA. The data was normally distributed and homogenous. The general characteristic of the subjects are described in table 1.
Table 1. General characteristic of the subjects.

\begin{tabular}{|c|c|c|c|}
\hline & Characteristic & $\begin{array}{l}\text { Bipolar } \\
\text { Hemiarthroplasty }\end{array}$ & PFNA \\
\hline \multirow[b]{2}{*}{ Sex } & Male & $8(23.53 \%)$ & $26(38.23 \%)$ \\
\hline & Female & $26(76.47 \%)$ & $42(61.77 \%)$ \\
\hline \multirow{3}{*}{ Age } & Range (year) & $36-87$ & $29-93$ \\
\hline & Mean (year) & $70.53 \pm 11.5$ & 70.19 \\
\hline & & & 12.25 \\
\hline \multirow{2}{*}{$\begin{array}{l}\text { Length of } \\
\text { Stay }\end{array}$} & Range (year) & $6-40$ & $4-39$ \\
\hline & Mean (year) & $15.59 \pm 8.38$ & $12.38 \pm 6.45$ \\
\hline \multirow[b]{2}{*}{ Mortality } & Dead & $7(20.59 \%)$ & $7(10.29 \%)$ \\
\hline & Alive & $27(79.41 \%)$ & 61 (89.71\%) \\
\hline \multirow{4}{*}{$\begin{array}{l}\text { Fracture } \\
\text { Type }\end{array}$} & 1 & 0 & 5 \\
\hline & 2 & 22 & 47 \\
\hline & 3 & 9 & 7 \\
\hline & 4 & 3 & 9 \\
\hline
\end{tabular}

PFNA: proximal femoral nail-antirotation
In comparing the mortality proportion between both groups, the subjects whose age over 60 years old were excluded to avoid any bias related to mortality cause so there were 28 cases of bipolar hemiarthroplasty and 58 cases of PFNA analyzed. In the bipolar hemiarthroplasty group, there were 6 deaths out of 28 subjects whereas in the PFNA group, there were 6 deaths out of 58 cases. The mortality proportion for each group was calculated by dividing the death number by the total subjects in each group. The chi-square test with $\alpha=0.05$, cross tabulation analysis, and odd ratio (OR) calculation were then performed to compare the mortality proportion between each group and to assess the significance of both procedures as risk factor of mortality in patients with intertrochanteric femur fracture. There was a significant difference in mortality proportion between the bipolar hemiarthroplasty group $(21.4 \%)$ and the PFNA group $10.3 \%(p=0.028)$ with OR 4.105 (95\%Cl 1.288-13.082).

Table 2. Mortality rate and fixation type

\begin{tabular}{|c|c|c|c|c|}
\hline & Dead & Alive & p & $\begin{array}{c}\text { OR } \\
(95 \% \mathrm{Cl})\end{array}$ \\
\hline $\begin{array}{c}\text { Bipolar } \\
\text { Hemiarthroplasty }\end{array}$ & $6(21.4 \%)$ & $\begin{array}{c}22 \\
(78.6 \%)\end{array}$ & & $\begin{array}{r}4.105 \\
(1.288-\end{array}$ \\
\hline PFNA & $6(10.3 \%)$ & $\begin{array}{c}52 \\
(89.7 \%)\end{array}$ & 0.020 & 13.082) \\
\hline
\end{tabular}

PFNA: proximal femoral nail-antirotation, LoS: Length of Stay

In comparing the proportion of long length of stay (long LoS) between both groups, there were 34 subjects treated with bipolar hemiarthroplasty and 68 subjects treated with PFNA. In the bipolar hemiarthroplasty group, there were 17 out of 34 subjects (50\%) who had long LoS ( $\geq 14$ days) whereas in the PFNA group, there were 22 out of 68 subjects (32.4\%) who had long LoS. The chisquare test with $\alpha=0.05$ was performed to compare the proportion of long LoS between each group. The difference of long LoS proportion between both groups was not statistically significant $(p=0.130)$. 
Table 3. Length of stay and fixation type

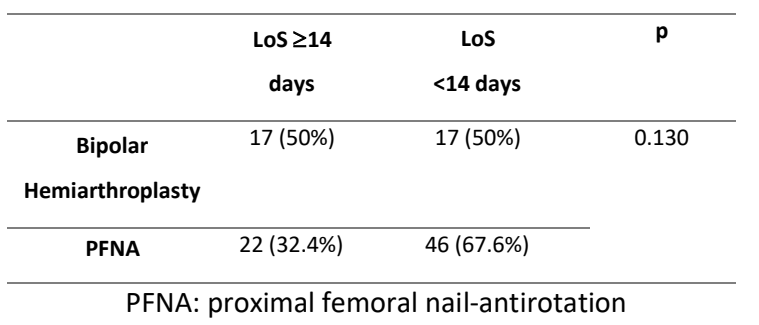

In assessing the proportion of long length of stay (long LoS) as risk factor of mortality in patients with intertrochanteric femur fracture, the subjects whose age over 60 years old were excluded to avoid any bias related to mortality cause so there were total of 86 subjects studied. Cross tabulation analysis was performed which long LoS was treated as risk factor for mortality and then, chi-square test with $\alpha=0.05$ was performed to compare the mortality proportion between subjects with long LoS and subjects without long LoS. There were 4 out of 29 (13.8\%) deaths in the subjects with long LoS whereas there were 8 out of $57(14.1 \%)$ deaths in the subjects without long LoS. The difference of mortality proportion between both groups was not statistically significant $(p=0.976)$.

Table 4. LoS: Length of Stay

\begin{tabular}{lccc}
\hline & Dead & Alive & p \\
\cline { 1 - 2 } LoS $\geq 14$ days & $4(13.8 \%)$ & $25(86.2 \%)$ & 0.976 \\
& & & \\
\cline { 1 - 2 } LoS $<14$ days & $8(14.1 \%)$ & 49 & \\
& & $(85.9 \%)$ &
\end{tabular}

\section{Discussion}

In this study, we founded that the majority of subjects who had intertrochanteric femur fracture were female with mean age of approximately 70 years old, and Boyd-Griffin type2 fracture was the most commonly found. This result may be related to the pathophysiology of intertrochanteric femur fracture which there are increased of fragility in the proximal part of the femur related to osteoporosis, so epidemiologically, intertrochanteric femur fractures are commonly founded in geriatric population with the proportion of men and women is $1: 4 .^{2}$ Beside that, we also found that the mean LoS were 12 to 15 days and the mortality proportion of the patients with intertrochanteric femur fracture treated with bipolar hemiarthroplasty and PFNA in two years postoperatively was $13.72 \%$.

Lim et al. reported that patients who underwent bipolar hemiarthroplasty after femoral neck fractures have 1-year mortality rate of $11.2 \%$, approximately 2.7 times higher than general population who are in same age group. ${ }^{9}$ In a separate study with similar population, Şahin et al. reported that patients who underwent PFNA after intertrochanteric fractures have 1-year mortality rate of $9.5 \% .{ }^{10}$ Hence in our center, it was still plausible to do any of those two fixations for intertrochanteric fractures, regardless the fracture type.

Based on two-years post-operative mortality proportion analysis in our center, this study showed that there was a significant difference in 2-year mortality rate between the bipolar hemiarthroplasty group (21.4\%) and the PFNA group $(10.3 \%)(p=0.028)$. This result was similar with a study conducted by Huang et al found that the 1-year mortality rate of intertrochanteric femur fracture patients whose age over 80 years old and was treated with bipolar hemiarthroplasty was significantly higher than those who was treated with PFNA. ${ }^{11}$ But the other studies found slightly different results. A study conducted by Esen et al showed that there was no significant difference in 1-year mortality rate between patients with intertrochanteric femur fracture with mean age of 79 years old treated with bipolar hemiarthroplasty compared with those treated with PFNA, although there were 5 cases of intraoperative mortality reported in subjects treated with bipolar hemiarthroplasty, and there was no intraoperative mortality reported in subjects treated with PFNA. ${ }^{12}$ Similar result was also obtained by Luo et al who found that there was also no significant differences, albeit higher, in 1-year mortality rate between intertrochanteric femur fracture patients with mean age of 80 years old treated with bipolar hemiarthroplasty compared to those who was treated with PFNA. ${ }^{6}$

In this study, we found that in bipolar hemiarthroplasty group, there were $50 \%$ subjects 
who had long LoS, whereas in the PFNA group, there were $32.4 \%$ subjects who had it. The difference of LoS in both group was not statistically significant $(p=0.13)$. Study by Luo et al showed different result. His study showed that in patients with intertrochanteric femur fracture with mean age of 80 years old, the LoS in the patients treated with bipolar hemiarthroplasty was significantly higher compared to those who was treated with PFNA. PFNA procedure is less invasive so it allows faster recovery, early weightbearing, early discharge, and associated with less complications. ${ }^{6}$

This study also showed that the mortality proportion in two years post-operative period was $13.8 \%$ in subjects who had long LoS and $14.1 \%$ in subjects without long LoS. The difference of LoS between both groups was non statistically significant $(p=0,976)$ so we did not find any relation between the long LoS and mortality proportion in this study. This result was different with study conducted by Lingsma et al about the relation of LoS with general mortality rate in hospital, in which there was significant difference in general mortality rate between patients with long LoS compared to those without long LoS. ${ }^{13}$

Nordström et al conducted a study about hip fractures in geriatric patients, which showed that for patients with length of stay of $\leq 10$ days, each 1-day reduction in length of stay will increase the odds of death within 30 days of discharge by $8 \%$ (odds ratio 1.08) in 2006, which even increased to $16 \%$ in 2012 (odds ratio 1.16). ${ }^{14}$ But it was in contrast with similar study by Nikkel et al which showed that in geriatric patient with hip fractures treated operatively, the length of stay more than 14 days increased the mortality rate significantly to $103 \% .{ }^{15}$ In our hospital, the length of stay has the range of 4 until 40 days, in which it might be attributed equally both due to patient condition, and also hospital situation which sometimes was overloaded.

\section{Conclusions}

Patients with intertrochanteric fracture who underwent bipolar hemiarthroplasty procedure have higher risk of 2-year mortality, but do not have higher risk to experience long stay at hospital (14 days or more) compared to ones treated with PFNA. In this study, length-of-stay itself is not associated with increased mortality risk in patients with intertrochanteric fracture who underwent bipolar hemiarthroplasty or PFNA procedure. Future studies to quantify other possible mortality factor beside implant fixation was needed to further improve the 2-year survival rate of patients with intertrochanteric fracture.

\section{Conflict of Interest}

The authors affirm no conflict of interest in this study

\section{Acknowledgement}

None.

\section{References}

1. Zhou S, Liu J, Zhen P, et al. Proximal femoral nail anti-rotation versus cementless bipolar hemiarthroplasty for unstable femoral intertrochanteric fracture in the elderly: a retrospective study. BMC Musculoskelet Disord. 2019;20(1):500.

2. Chang SM, Hou ZY, Hu SJ, Du SC. Intertrochanteric Femur Fracture Treatment in Asia: What We Know and What the World Can Learn. Orthop Clin North Am. 2020;51(2):189-205.

3. Ülkü TK, Tok O, Seyhan M, Gereli A, Kaya A. Comparison of Third Generation Proximal Femoral Nails in Treatment of Reverse Oblique Intertrochanteric Fractures. Bezmialem Science. 2019;7(4):271-5.

4. Liu JJ, Shan LC, Deng BY, et al. Reason and treatment of failure of proximal femoral nail antirotation internal fixation for femoral intertrochanteric fractures of senile patients. Genetics and Molecular Research. 2014; 13(3): 5949-5956

5. Xie $Y$, Dong $Q$, Xie Z. Proximal femoral nail anti-rotation (PFNA) and hemi-arthroplasty in the treatment of elderly intertrochanteric fractures. Acta Orthop Belg. 2019;85(2):199-204.

6. Luo X, He S, Zeng D, Lin L, Li Q. Proximal femoral nail antirotation versus hemiarthroplasty in the treatment of senile intertrochanteric fractures: Case report. Int J Surg Case Rep. 2017;38:37-42.

7. Dusak WS, Simanjuntak HA, Putra IGNPW. Comparison between the results of proximal femur nail anti-rotation and 
cemented bipolar hemiarthroplasty in treatment of intertrochanteric fractures in Sanglah Hospital in 2016. Int J Res Med Sci. 2019;7(9): 3475-3479.

8. Doyle DJ, Goyal A, Bansal P, et al. American Society of Anesthesiologists Classification (ASA Class) [Updated 2020 Apr 7]. In: StatPearls [Internet]. Treasure Island (FL): StatPearls Publishing; 2020 Jan-. Available from:

https://www.ncbi.nlm.nih.gov/books/ NBK441940/

9. Lim YW, Kwon SY, Han SK, et al. Postoperative mortality and factors related to mortality after bipolar hemiarthroplasty in patients with femoral neck fractures. J Arthroplasty. 2009;24(8):1277-1280.

10. Şahin EK, İmerci A, Kınık $H$, et al. Comparison of proximal femoral nail antirotation (PFNA) with AO dynamic condylar screws (DCS) for the treatment for unstable peritrochanteric femoral fractures. Eur J Orthop Surg Traumatol. 2014; 24:347-352.

11. Huang J, Shi Y, Pan W, et al. Bipolar Hemiarthroplasty should not be selected as the primary option for intertrochanteric fractures in elderly patients. Sci Rep. 2020;10(1):4840.

12. Esen $E$, Dur $H$, Ataoğlu MB, Ayanoğlu $T$, Turanlı S. Evaluation of proximal femoral nail-antirotation and cemented, bipolar hemiarthroplasty with calcar replacement in treatment of intertrochanteric femoral fractures in terms of mortality and morbidity ratios. Eklem Hastalik Cerrahisi. 2017;28(1):35-40.

13. Lingsma HF, Bottle A, Middleton S, Kievit J, Steyerberg EW, Marang-van de Mheen PJ. Evaluation of hospital outcomes: the relation between length-of-stay, readmission, and mortality in a large international administrative database. $B M C$ Health Serv Res. 2018;18(1):116.

14. Nordström P, Gustafson Y, Michaëlsson K, Nordström A. Length of hospital stay after hip fracture and short term risk of death after discharge: a total cohort study in Sweden. BMJ. 2015;350:h696.

15. Nikkel LE, Kates SL, Schreck M, et al. Length of hospital stay after hip fracture and risk of early mortality after discharge in New York state: Retrospective cohort study. BMJ 2015;351:h6246. 